\title{
Una aproximación a la función de los proyectos tecnológicos de la agricultura familiar en el distrito de Matucana, Huarochirí, Lima.
}

\author{
Catherine Alva ${ }^{1}$, Melchorita Castro², Merely Tumbalobos ${ }^{3}$ \\ ${ }^{1}$ Universidad Nacional Agraria La Molina, Lima, Perú \\ 2,3 Universidad Nacional Mayor de San Marcos, Lima, Perú \\ 1,2,3 International Terraced Landscapes Alliance, Lima, Perú
}

Enviado el 15 de noviembre del 2021. Aceptado el 8 de enero del 2022

DOI: https://doi.org/10.33017/RevECIPeru2021.0008/

\section{Resumen}

Para establecer la función que cumplen los proyectos tecnológicos en la agricultura familiar en el distrito de Matucana, se ha utilizado información del IV Censo Nacional Agropecuario, el Sistema Integrado de Estadística Agraria, normativas del Ministerio de Agricultura, y entrevistas a actores locales y expertos en la materia. Se realizaron entrevistas de forma remota. Se evidencia que su agricultura familiar está constituida por comunidades campesinas y asociación de productores y es eminentemente montañosa, donde más de la mitad de la superficie agrícola está conformada por andenes y administrada principalmente por varones. La cercanía a la ciudad de Lima determina los cultivos que se producen y genera la necesidad de una mejora constante de su calidad. Asimismo, el uso de las tecnologías ancestrales y modernas es relevante y ampliamente aceptado. Se han ejecutado diferentes proyectos por el Estado y ONGs. Sin embargo, se demuestra que la comunidad campesina tiene sus propias iniciativas de adopción tecnológica debido a que los proyectos implementados son insuficientes. En ese sentido, se recomienda que la academia centre sus investigaciones en las tecnologías ancestrales que aún no han sido reconocidas pero que son utilizadas por las comunidades campesinas.

Descriptores: proyecto tecnológico, agricultura familiar, zona montañosa, tecnología ancestral, tecnología moderna.

\section{Abstract}

To establish the role of technological projects in family farming in the Matucana district, information from the IV National Agricultural Census, the Integrated System of Agrarian Statistics, regulations of the Ministry of Agriculture, and interviews with local actors and experts in the field have been used. Interviews were conducted remotely. It is evident that their family farming is made up of rural communities and producers association, and is eminently mountainous, where more than half of the agricultural area is made up of terraces and administered mainly by men. The proximity to the city of Lima determines the crops that are produced and generates the need for constant improvement of their quality. Likewise, the use of ancient and modern technologies is relevant and widely accepted. Different projects have been performed by the State and NGOs. However, it is shown that the rural community has its own technology adoption initiatives because the projects implemented are insufficient. In this sense, it is recommended that the academy focus its research on ancient technologies that have not yet been recognized but are used by rural communities.

Keywords: technological project, family farming, mountainous area, ancient technology, modern technology. 


\section{Introducción}

La tecnología que se implementa en la agricultura familiar de países de montaña mediante proyectos abarca desde las modernas hasta las de origen ancestral. La agricultura familiar es la forma de agricultura predominante y un estilo de vida extendido tanto en países desarrollados como en desarrollo, constituida por pequeños y medianos productores, está comprendida por campesinos, pueblos indígenas, agricultores de la zona montañosa, pastores y muchos otros [1].

Los especialistas en tecnologías para la agricultura familiar señalan diferentes recomendaciones sobre las condiciones para su mejor implementación en aspectos técnicos, económicos y de políticas públicas. Sin embargo, con menos frecuencia se encuentran estudios sobre la función que cumplen los proyectos tecnológicos en el desarrollo de la agricultura familiar conformada por comunidades campesinas tradicionales de la zona montañosa.

Una de las bondades de la tecnología es que permite contrarrestar la escasez de mano de obra y el encarecimiento del jornal causado por la emigración rural. Estos problemas han motivado a que el pequeño agricultor del Asia use tecnologías que le ayuden a manejar el agua, suelo y energía de forma más eficiente [2], además, una agricultura verde, recreativa, turística y de alta productividad [3]. En las montañas de Nepal emplean motocultivadores, en especial en los cultivos de labor intensiva como el arroz, logrando aumentar su productividad, mejorar el pago del jornal y disminuir el trabajo de los animales de tiro [4].

Los factores que determinan la adopción de tecnologías modernas están asociados a los bienes, crédito disponible, electrificación y disponibilidad de caminos que permiten un ambiente adecuado para la adopción progresiva de equipos y maquinaria agrícola a pequeña escala [5]. En sentido, las políticas agrarias deben priorizarse para el pequeño agricultor a través de infraestructura física, servicios y asistencia técnica sólida [5], [6].

En la experiencia europea y asiática se puede mencionar los programas y proyectos de concentración de parcelas, distinguiéndose el logro alcanzado por los países de Europa occidental, China y Japón mediante políticas sólidas donde la participación de los actores locales -en especial de los pequeños agricultores - ha sido fundamental en la elaboración de los planes de los proyectos implementados [7], [8].

En la zona altoandina se proponen un conjunto de tecnologías y prácticas para la conservación de servicios ecosistémicos, entre las se tiene el barbecho, enmiendas del suelo, labranza de conservación, pastoreo rotativo, restauración ecológica de pastizales, conservación de la agrobiodiversidad, riego moderno, entre otros [9].

Mientras, en la pequeña agricultura latinoamericana [10] y en la zona andina de Ecuador [11] y Perú [10], [12] se fomenta la adopción de tecnologías en manejo integrado de plagas, la agricultura orgánica y agroecología para controlar problemas de plagas y el alto uso de pesticidas.

Otra tecnología que se implementa en la agricultura familiar Latinoamericana y la zona rural andina es el biogás [13], [14], mientras la energía solar fotovoltaica es una tecnología prometedora [15].

Mediante los proyectos tecnológicos en la zona rural se busca mejorar la infraestructura y los servicios que recibe el agricultor familiar para mejorar su producción, acceder al mercado y aumentar sus ingresos económicos.

En el Perú, la agricultura familiar (AF) es el modo de vida y de producción realizada por varones y mujeres del mismo núcleo familiar a cargo de diversos sistemas productivos en un mismo territorio. Contempla la producción agrícola, pecuaria, forestal, industrial rural, pesca artesanal, acuicultura y otros [16]. Clasificada en AF de subsistencia (AFS) con unidades agropecuarias familiares (UA) menores a 2 hectáreas estandarizadas, la AF intermedia (AFI) con UA entre 2 y 5 hectáreas y la consolidada (AFC) entre 5 y menores a 10 hectáreas [17].

En la comunidad campesina del distrito de Matucana, provincia de Huarochirí, se implementan tecnologías modernas como el riego, agricultura orgánica, mecanización [18], infraestructura de riego y riego tecnificado, así como las de origen ancestral como la rehabilitación de andenes, construcción de cochas y zanjas de infiltración [19], [20].

Sin embargo, a pesar de los proyectos ejecutados y avances de la agricultura familiar en Matucana, no se ha alcanzado el desarrollo esperado. Por ese motivo, en este artículo se elabora una reflexión que 
busca aproximarse a la función que cumplen los proyectos tecnológicos en la agricultura familiar de Matucana con base en los juicios de expertos y el testimonio de la autoridad comunal local, como paso previo a la elaboración de los instrumentos de campo para recoger información desde los actores locales.

\section{Metodología}

\subsection{Agricultura familiar en Matucana:}

Matucana es la capital de la provincia de Huarochirí. Se encuentra a $74.5 \mathrm{~km}$ de la ciudad de Lima. Su altitud es entre 2500 a 4500 msnm, en la Subcuenca Santa Eulalia-Parac, cuenca del Rímac. Presenta una temperatura media anual de $15.4^{\circ} \mathrm{C}$, precipitación media anual $285.2 \mathrm{~mm}$ y humedad relativa media anual $70 \%$ [21].

Para el 2017 tenía una población de 4508 habitantes [22]. En el distrito habitan 3 comunidades campesinas: Barrio Alto de Matucana, Barrio Bajo de Matucana y Huariquiña [23]. Para el 2012 contaba con 366 productores agropecuarios, de los cuales el $71.6 \%$ son varones y $28.4 \%$ mujeres [24]. Además, el $94.3 \%$ (345 productores) se encuentra calificado como agricultor familiar [25]. El 30.3\% se encuentra entre 20 a 49 años, $47.0 \%$ entre 50 a 69 y $22.7 \%$ de 70 a más (ver Figura 1).

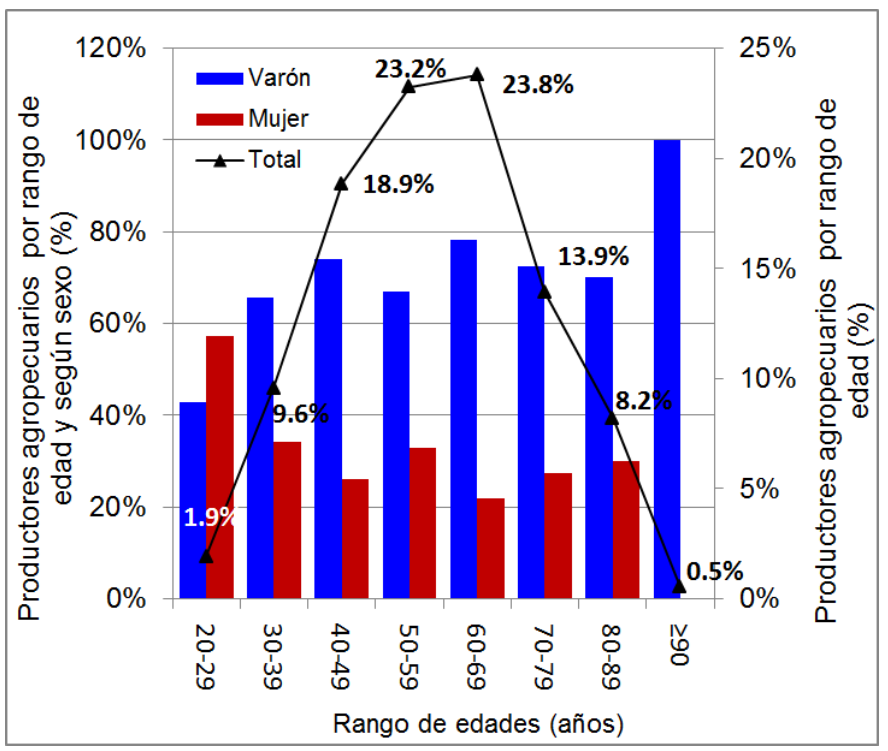

Figura 1: número de productores agropecuarios en porcentaje (eje de abscisas) distribuidos por rango de edades en años (eje de ordenadas), según sexo (varones-barra azul y mujeres-barra guinda). Datos tomados de la referencia [24].
Así también, de los productores agropecuarios varones el $66.8 \%$ son agricultores y el $3.8 \%$ ganaderos; mientras, de las productoras mujeres el $72.1 \%$ son agricultoras y $2.9 \%$ ganaderas (ver Tabla 1).

Tabla 1: profesión u oficio del productor agropecuario por sexo y porcentaje. Datos tomados de la referencia [24].

\begin{tabular}{|l|c|c|c|c|}
\hline $\begin{array}{c}\text { Prof./Oficio } \\
\text { del productor } \\
\text { agropecuario }\end{array}$ & Varón & $\%$ & Mujer & $\%$ \\
\hline $\begin{array}{l}\text { Agrónomos y } \\
\text { afines }\end{array}$ & 2 & 0.8 & 0 & 0.0 \\
\hline Técnicos & 29 & 11.1 & 8 & 7.7 \\
\hline Agricultor & 175 & 66.8 & 75 & 72.1 \\
\hline Ganadero & 10 & 3.8 & 3 & 2.9 \\
\hline Otros & 46 & 17.6 & 18 & 17.3 \\
\hline \multicolumn{1}{|c|}{ Total } & 262 & 100.0 & 104 & 100.0 \\
\hline
\end{tabular}

Con respecto a la superficie agrícola en Matucana, asciende a 2506.0 ha [26]. Asimismo, para el 2012, se inventarió 1968.1 ha de andenes de los cuales el $78.3 \%$ se encontraba en uso y $21.7 \%$ en abandono [27]. Como se puede ver en la Tabla 2 , el $59.7 \%$ y $37.6 \%$ de andenes inventariados se encuentran sobre pendientes entre $25-50 \%$ y $50-75 \%$, respectivamente.

Tabla 2: distribución de andenes (ha) por rangos de pendiente (\%). Datos tomados de la referencia [27].

\begin{tabular}{|c|c|c|c|c|c|c|}
\hline $\begin{array}{l}\text { Pend } \\
.(\%)\end{array}$ & $\frac{10}{v}$ & $\begin{array}{l}\text { 足 } \\
\text { ஸे }\end{array}$ & $\begin{array}{l}\text { 우 } \\
\text { ట్ }\end{array}$ & 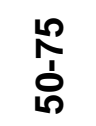 & $\stackrel{n}{\Lambda}$ & Total \\
\hline (ha) & $\begin{array}{l}7 . \\
7\end{array}$ & $\begin{array}{c}19 . \\
9\end{array}$ & $\begin{array}{c}1175 . \\
7\end{array}$ & $\begin{array}{c}740 . \\
7\end{array}$ & $\begin{array}{c}24 . \\
1\end{array}$ & $\begin{array}{c}1968 . \\
1\end{array}$ \\
\hline (\%) & $\begin{array}{l}0 . \\
4\end{array}$ & 1.0 & 59.7 & 37.6 & 1.2 & 100 \\
\hline
\end{tabular}

En tanto, la agricultura familiar (AF) del distrito está constituida en $32.0 \%$ por la AFS, $55.0 \%$ AFI y $13.0 \%$ AFC [25]. Además, como se observa en la Figura 2, la mayor cantidad de las UA de la AF matucanence es administrada por varones (barras azul y celeste), quienes tienen a cargo el $39.0 \%$ de AFS, $34.0 \% \mathrm{AFI}$ y $36.0 \%$ AFC con cultivos, mientras las mujeres el $11.0 \%, 10.0 \%$ y $6.0 \%$, respectivamente (ver Figura 2). 


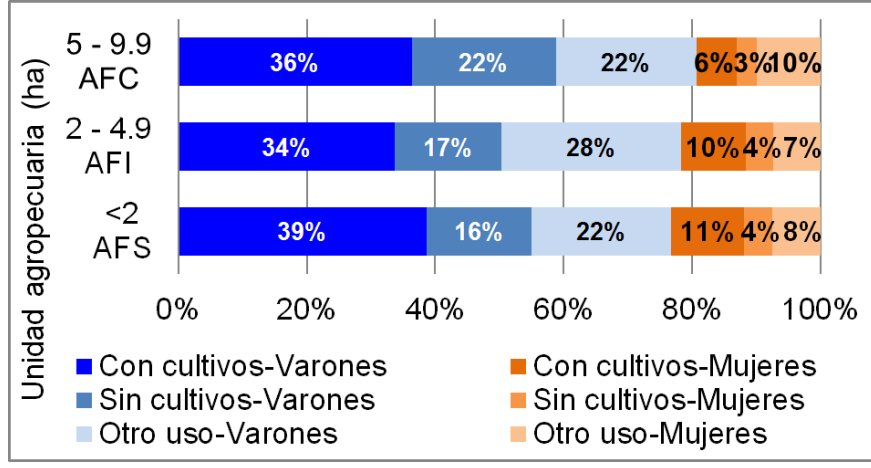

Figura 2: uso de la tierra con cultivos, sin cultivos y otros usos administrados por varones (barras azules y celestes) y mujeres (barras naranjas). Datos tomados de la referencia [24].

En cuanto a la superficie con cultivos, para el 2012, bajo riego se tenía 1119.8 ha y bajo secano 1.1 ha. Asimismo, como se puede observar en la Figura 3, el destino de los cultivos bajo riego principalmente son $77.4 \%$ para la venta, $15.5 \%$ para consumo.

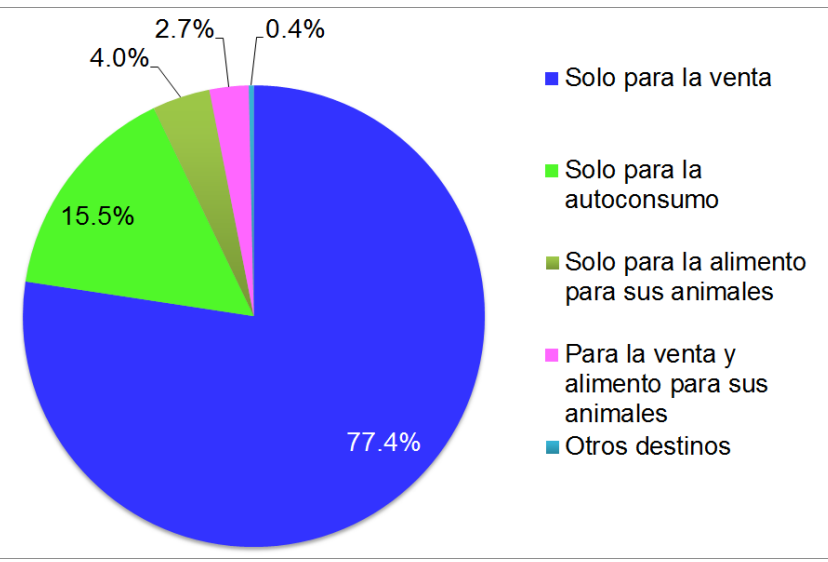

Figura 3: destino de la mayor parte de la producción bajo riego en porcentaje. Datos tomados de la referencia [24].

Los principales cultivos bajo riego para el 2012, eran la alfalfa con 357.8 ha, palto 210.3 ha, papa blanca 187.5 ha, chirimoyo 102.6 ha, trigo 102.3 ha, maíz choclo 102.2 ha; $y$, bajo secano, como único cultivo está la tuna con 1.1 ha [24].

Mientras que la intención de siembra para la Campaña Agrícola agosto 2018 - julio 2019, se tenían 12 ha de arveja grano verde, maíz amiláceo 10 ha, maíz choclo 8 ha, papa (blanca) 8 ha y papa color 8 ha [28]. Y, para el campaña agosto 2019 Julio 2020 la arveja grano verde 10 ha, maíz amiláceo 10 ha, maíz choclo 12 ha y papa blanca 12 ha [29].
En cuanto al uso de tractores en las actividades agropecuarias de la AF solo 2 varones y 2 mujeres productoras agropecuarias manifestaron utilizarlos [24]. Mientras el uso de energía animal es más utilizado en la AFS con $43.3 \%$, le sigue la AFI $7.6 \%$ y AFC $2.3 \%$ (ver Figura 4 ).

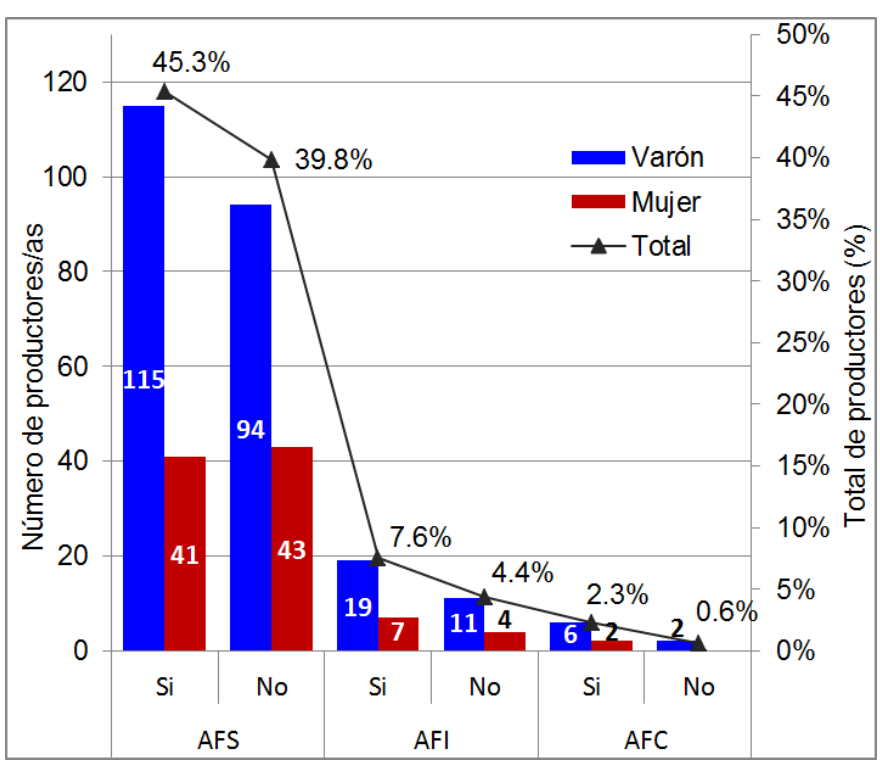

Figura 4: energía animal utilizada por los productores agropecuarios, clasificados por tipología de $A F$ y sexo (varones-barra azul y mujeres-barra guinda). Datos tomados de la referencia [24].

Sobre las condiciones de la actividad agropecuaria, para el 2012, 22 productores tenían animales de raza, 61 contaban con asistencia técnica o capacitación, 44 usaban semilla certificada y 326 estaban asociados o formaron parte de la comunidad campesina [25].

\subsection{Enfoque y recopilación de datos:}

Se ha utilizado el enfoque cualitativo y cuantitativo, ya que permite recolectar, analizar y vincular datos desde ambas perspectivas. El tipo de recopilación aplicada ha sido la documental y campo, que permite consolidar los datos y resultados obtenidos. Se inicia con la información documental para los antecedentes y se sigue con el diseño de métodos e instrumentos de investigación [30].

Las entrevistas han sido de información y dirigida. La primera ayuda a obtener datos en la investigación científica y definir metodologías y técnicas de investigación mediante la entrevista a académicos e investigadores quienes brindan valiosos consejos, sugerencias y comentarios que ayudan a conocer más sobre la investigación. Las 
entrevistas dirigidas se enfocan en las opiniones del entrevistado siguiendo un guion preestablecido [30].

\subsection{Selección de los entrevistados:}

Para la selección de los entrevistados se consideró aspectos académicos, técnicos y la experiencia local, nacional e internacional en agricultura familiar. En representación de la experiencia local, se seleccionó a Richard Melo, actual presidente de la comunidad campesina de Barrio Bajo de Matucana, donde se han realizado proyectos con tecnologías ancestrales y modernas. Así también, a Iván Medina, técnico de AgroRural, quien ha participado de proyectos tecnológicos en Matucana y otros distritos de Huarochirí.

En representación del Estado a Mirbel Epiquién, quien tiene a cargo una jefatura nacional en AgroRural. Por parte de la academia y el enfoque cultural a Nilda Varas, por el enfoque de género a Fánel Guevara, por lo técnico-agronómico a Carmen Felipe-Morales y desde la experiencia de la cooperación internacional a José Benites (ver Tabla $3)$.

Tabla 3: Relación de entrevistados por nombres y cargos o especialidades. Elaboración propia.

\begin{tabular}{|c|l|l|}
\cline { 2 - 3 } \multicolumn{1}{|c|}{$N^{\circ}$} & \multicolumn{1}{c|}{ Nombre } & \multicolumn{1}{c|}{ Cargo o Especialidad } \\
\cline { 2 - 3 } 1 & Richard Melo & $\begin{array}{l}\text { Presidente de la } \\
\text { comunidad campesina de } \\
\text { Barrio Bajo de Matucana. }\end{array}$ \\
\hline 2 & Iván Medina & $\begin{array}{l}\text { Técnico de AgroRural, } \\
\text { residente en Matucana. }\end{array}$ \\
\hline 3 & $\begin{array}{l}\text { Mirbel } \\
\text { Epiquién }\end{array}$ & $\begin{array}{l}\text { Jefe de la Unidad de } \\
\text { Cadena de Valor } \\
\text { Agroforestal, Forestal y } \\
\text { Silvo Pastoril de } \\
\text { AgroRural-MIDAGRI. }\end{array}$ \\
\hline 4 & Nilda Varas & $\begin{array}{l}\text { Antropóloga. Doctora en } \\
\text { Estudios Ambientales. }\end{array}$ \\
\hline 5 & Fánel Guevara & $\begin{array}{l}\text { Experta en enfoque de } \\
\text { género. }\end{array}$ \\
\hline 6 & $\begin{array}{l}\text { Carmen } \\
\text { Felipe-Morales }\end{array}$ & $\begin{array}{l}\text { Doctora en Ciencias } \\
\text { Agronómicas. }\end{array}$ \\
\hline 7 & José Benites & $\begin{array}{l}\text { Experto en cooperación } \\
\text { internacional. }\end{array}$ \\
\hline
\end{tabular}

\subsection{Elaboración de guiones:}

Se elaboró una guía para cada entrevistado en función a su cargo o especialidad y de los objetivos específicos de la investigación: 1- Describir las características de la agricultura familiar en
Matucana. 2- Identificar las características de los proyectos tecnológicos de la agricultura familiar en Matucana. 3- Determinar la relación que existe entre los proyectos tecnológicos y la agricultura familiar en Matucana.

\subsection{Entrevistas personales:}

Para las entrevistas se contactó previamente con los entrevistados por medio de llamada y/o correo electrónico para acordar la fecha y hora. Las entrevistas se realizaron entre el 19 al 28 de octubre de 2021, de forma remota, tanto por Zoom, Google Meet y llamada telefónica. Todas fueron grabadas y transcritas para su análisis.

\subsection{Limitaciones:}

En este artículo se elabora una reflexión que busca aproximarse a la función que cumplen los proyectos tecnológicos en la agricultura familiar matucanence con base en juicios de expertos, ya que se busca obtener sus consejos, sugerencias y comentarios que ayuden a reconocer los tópicos que debemos incluir en la investigación. Asimismo, por la pandemia, las limitaciones se observan en las entrevistas a los productores agropecuarios de Matucana. Sin embargo, con las evidencias encontradas se espera poder elaborar instrumentos de campo que permitan recoger de mejor forma la información desde los actores locales.

\section{Resultados y Discusión de resultados}

\subsection{Agricultura familiar en Matucana}

La agricultura familiar engloba diversas actividades productivas, como las agropecuarias, forestales, entre otras. Se emplea la mano de obra familiar y su producción se destina a su seguridad alimentaria, así como para generar ingresos que le permitan invertir en su producción [31]. En el Perú existe una vasta variedad de agriculturas; entre más próximas a la costa está dotada de mejor infraestructura, como carreteras, energía eléctrica, acceso a mercados, etc., a diferencia de las que se ubican a mayor altitud. Inclusive, si solo analizamos la provincia de Huarochirí, donde se ubica el distrito de Matucana, encontraremos una diversidad de agriculturas familiares, ya que esta provincia incluye diferentes altitudes [32], desde la costa hasta la janca, dentro de las cuencas que abarca.

En el entorno de la comunidad campesina, esta agricultura depende no solo de la familia, sino de la ayuda mutua para garantizar su producción [34]. 
Esta agricultura también se caracteriza por no dedicarse exclusivamente a actividades agropecuarias. Su fuente de ingresos se compone además de otras labores como el comercio o empleos temporales en la ciudad [33], [34]. De esta manera, la economía familiar "ya no solo funciona en relación con lo comunal, sino también con lo urbano" [33].

La dinámica de la migración está cambiando la agricultura familiar. En el entorno rural, no se han cubierto la totalidad de necesidades básicas a comparación del entorno urbano [34]. Entonces, se ha generalizado la idea de que el incremento en la calidad de vida es directamente proporcional a obtener educación superior y vivir en la ciudad. De esta manera, los jóvenes se alejan de las labores agrícolas y se altera la línea de transmisión de saberes [33].

Al cambiar la producción de cultivos nativos hacia una producción predominantemente para la venta [34], el autoconsumo se ha reducido [33], [34], lo que genera cambios en su dieta alimentaria. El uso del suelo agrícola también se ha transformado para dedicarlo a actividades que generan más ingresos inmediatos, como la venta. En su defecto, estas tierras son abandonadas. De esta manera "los espacios de cultivo se han ido reduciendo y con esto, las actividades diversas que tenía el campo" [34].

Esta situación pone en riesgo a la agricultura familiar [33], que se caracteriza por ser principalmente administrada por los miembros de la familia [35]. Sin embargo, también representa una oportunidad, puesto que los jóvenes que retornan lo hacen con conocimientos adquiridos en las tecnologías de la información, en la dinámica comercial o en procesos en la cadena de valor, que pueden ser aplicados en la mejora de la eficiencia y productividad en el campo [34].

La agricultura familiar en Matucana representa más del $90.0 \%$ de la agricultura en la zona. De este modo, más de la mitad de la agricultura familiar matucanense es del tipo intermedia (AFI) [25]. La compone esencialmente con $60.7 \%$ productores mayores de 50 años y es administrada principalmente por varones, quienes mantienen entre el 64.0 al $69.0 \%$ de las unidades agropecuarias con cultivo, mientras que las mujeres solo entre 11.0 a $6.0 \%$ (ver Figura 1 ).

La baja cantidad de unidades agropecuarias bajo la administración de las mujeres estaría asociada a la carga doméstica, en cuanto a alimentación, cuidado de niños y ancianos, limpieza, entre otras actividades que las mujeres realizan, además de la tenencia de tierra.

La superficie agrícola matucanense está conformada en un 78.5\% por andenes que se ubican en las laderas de la Sub-cuenca Santa Eulalia-Parac, lo que le confiere, además, la característica de ser una agricultura familiar de montañas. Los andenes en un $98.5 \%$ se encuentran en laderas con pendientes por encima del $25 \%\left(14^{\circ}\right)$ de inclinación (ver Tabla 2). Asimismo, casi toda la superficie agrícola cultivada se encuentra bajo riego y tiene como destino principal la venta (ver Figura 3).

En la agricultura familiar de Matucana se involucran todos los miembros de la familia, los cuales viven en el pueblo y diariamente se transportan hacia los anexos para realizar sus labores [36]. La comunidad campesina de Barrio Bajo de Matucana se dedica a la floricultura y al cultivo de tubérculos para el autoconsumo [37].

Richard Melo, presidente de la comunidad campesina de Barrio Bajo de Matucana, manifiesta que "hace 30 años atrás, la comunidad ofrecía papa a todos los mercados de Lima. Matucana era el mayor productor de papa por sus amplios terrenos y andenería"; sin embargo, debido a la importación de este tubérculo, los costos de producción no justifican los precios de venta para el productor local, por lo que se ha optado por la producción de flores para cubrir los costos de la canasta familiar [37].

Esta agricultura se enfrenta a una necesidad de producir para satisfacer la demanda del mercado [33], [34]. La producción de la agricultura familiar en Matucana está estrechamente ligada al acceso al mercado en la ciudad de Lima [33], [36], [37]. Es decir, la variedad de productos cultivados depende de su rentabilidad. Por este motivo, se ha reducido la diversidad de productos nativos cultivados como la quinua, maca y mashua en las partes altas o la diversidad de papas [34], [37].

La producción de la agricultura familiar de Matucana se comercializa en los mercados de Lima Metropolitana como Acho y Santa Anita. La negociación se realiza sin intermediarios, pues los agricultores son los que se encargan del transporte hacia la ciudad, entregan el producto y reciben el pago. La emigración se ha direccionado a las zonas donde comercializan sus productos, como Santa 
Anita y Ate Vitarte [36], estableciendo doble domicilio entre Matucana y estos distritos limeños [34].

\subsection{Proyectos tecnológicos en la agricultura familiar matucanence}

El concepto de tecnología es concebido desde lo cultural, en cuanto a la expresión del espíritu para el desarrollo tecnológico, y lo instrumental, como aquello que sirve para fines determinados. El concepto instrumental de la tecnología se centra en la innovación, dejando de lado la creatividad y los usos que las personas le dan en su vida diaria, prevaleciendo el conocimiento científico, restringiéndose a una élite técnica o inventiva [38].

Hay una fuerte predominancia a cosificar todo lo referente a la tecnología, olvidando que ésta es toda creación realizada por el hombre.

"La tecnología es una creación humana, es una cuestión cultural. Todo lo que es creación humana es cultura. (...) Hay cultura material e inmaterial. Es decir, cultura es la tecnología, cultura es un azadón (...) Lo que pasa es que muchas veces se cosifica la tecnología como si fueran cosas, pero detrás de las cosas está la creación humana, está la concepción que se tiene sobre el entorno, sobre la naturaleza, los significados" [33].

En los últimos años los proyectos de inversión han incorporado procesos participativos junto con tecnologías de conservación de agua y suelo como andenes, cochas, zanjas de infiltración, bofedales, entre otros. A su vez, tecnologías modernas, como riego tecnificado, paneles solares para bombear agua del subsuelo, invernaderos y otras innovaciones en manejo de cultivos y semillas mejoradas [36]. Aunque otros expertos mencionan que los proyectos son "parciales, no tienen una visión integral, son puntuales, es decir, tienen muy poca duración y solo resuelven una parte del problema" [34].

Sin embargo, AgroRural, institución que posee 40 años de experiencia, manifiesta que ostenta una serie de tecnologías con comprobado funcionamiento; aunque no descarta las innovaciones tecnológicas [32]. En la experiencia de esta institución estatal, al generarse una simbiosis con las comunidades es posible que los proyectos se ejecuten pronto, ya que las comunidades saben lo que necesitan y no es necesario dilatar la intervención.

\begin{abstract}
"Hay una relación importante con la comunidad. Las comunidades se entienden muy bien y no es necesario decirles lo que tienen que hacer, saben. Entonces, cuando se llega, hay una simbiosis rápida, se priorizan lugares de intervención en función de los recursos y se trabaja. Eso es. No necesitamos de mucha parafernalia para esto. No necesitamos de una estrategia o de nuevos enfoques. (...) Hay que ir de frente a la planificación y a la acción" [32].
\end{abstract}

La tecnología en la agricultura familiar es un gran avance. En Matucana, como en otros distritos de Huarochirí, los jóvenes han vuelto a sus pueblos a causa de la pandemia del Covid-19. Sin embargo, cuando las restricciones se levanten ellos volverán a las ciudades, pero quienes se quedarán son sus padres y abuelos. En este caso, la tecnología moderna, como los motocutores, ordeñadores, mochilas fumigadoras y otros equipos, reducen los costos y compensa la falta de mano de obra, también las capacitaciones en buenas prácticas agrícolas, manufactura y planes de negocio [36]. Además, las tecnologías ancestrales que permiten la conservación de los recursos naturales base de la producción agropecuaria como son el agua, suelo y la biodiversidad.

Más allá de la tecnología es quién lo va usar, "ese es un actor que hay que tomar en cuenta" [33].

\subsection{Relación entre la agricultura familiar y los proyectos tecnológicos}

Es necesario hacer proyectos integrales a nivel de microcuencas a partir de la recuperación del conocimiento de las tecnologías ancestrales que van más allá de los andenes, cochas, zanjas de infiltración y amunas. Es decir, ampliar la visión cultural y no solo centrarlo en lo físico. Desde la academia, Andrés Alencastre, Julio Valladolid y Mario Tapia [34] y, desde la comunidad campesina Gregorio Ríos [39] y Carlos Milla [40] han identificado una vastedad de tecnologías ancestrales a parte de las mencionadas.

Además, los proyectos tienen que tomar en cuenta los roles que juegan los actores locales, mujeres, varones y jóvenes del campo, para identificar las actividades que todos puedan realizar para asegurar su participación.

En el caso de la mujer, tiene que tomarse especial atención en capacitaciones sobe manejo de 
tecnologías, derechos, manejo de asambleas, el comercio, la pequeña industria. Es decir, hay varias posibilidades para que las mujeres sean capacitadas e informadas, como también los varones. Y, en el caso de los jóvenes, en tecnologías y técnicas de la información hidrogeográfica, hidrogeológica y agroclimática; además, el comercio electrónico. Por eso, es necesario aprender a generar proyectos imaginativos para que sean interlocutores y ayuden en los proyectos a las comunidades [34], [41].

La organización comunal ha sido un factor clave para el éxito de los proyectos [33], [34], [41]. La participación de la comunidad en la formulación y la ejecución de los proyectos también ha determinado su efectividad, es así que resulta importante entender el rol de cada actor dentro de la agricultura familiar para garantizar una efectiva participación dentro de los proyectos tecnológicos. En los que viene realizando el Estado se están promoviendo e incluyendo la participación de las mujeres y jóvenes con buena aceptación local [36].

Es la misma población local que debe manifestar sus requerimientos y necesidades, en la medida que se cree una buena relación, con empatía, conversando y escuchando a la población [31], [32], [33], [34], [41]. En un trabajo multisectorial y articulado entre las autoridades locales comunales y el Estado [31].

En el distrito de Matucana, el Estado y ONGs implementan proyectos tecnológicos, no obstante, muchas veces el apoyo no es suficiente, por ese motivo los productores familiares no conformes organizan sus propias iniciativas con el objetivo de mejorar la calidad de su producción con la utilización de las tecnologías ancestrales y modernas.

"La experiencia que tenemos [los campesinos] es mejor (...) aunque la ciencia avanza, con los dos estamos trabajando, la tecnología ancestral es buena, hay buenas experiencias (...), no se puede dejar eso. (...) A nivel de comunidad nos preocupamos por mejorar la calidad del producto, mi comunidad ha comprado productos de mejoramiento de ganado para la inseminación artificial, también estamos trabajando con Cáritas, ellos nos dan charlas para el mejoramiento de la producción, siembra de agua, cosecha de agua. Siempre estamos preocupados en mejorar en nuestra comunidad (...) Ahora ya no usamos los abonos químicos sino los orgánicos, es diferente" [37].

\section{Conclusiones}

Para establecer la función de los proyectos tecnológicos que se ejecutan en la agricultura familiar matucanence, hemos analizado sus características y se ha encontrado que está constituida por comunidades campesinas y asociación de productores, es predominantemente de montañas donde más de la mitad de la superficie agrícola está conformada por andenes y administrada principalmente por varones.

Para identificar los proyectos que se vienen implementando y determinar la relación entre las comunidades y las instituciones que intervienen en esta localidad, se ha entrevistado a actores locales y expertos en la materia. De esta forma, se ha observado que la cercanía del distrito huarochirano a la ciudad de Lima, principal destino de su producción agropecuaria, determina los tipos de cultivos que en esta se producen y genera la necesidad de mejorar la calidad productiva de los cultivos que el agricultor familiar produce.

De esta forma, el uso de las tecnologías ancestrales y modernas es relevante y ampliamente aceptado en la agricultura familiar matucanense. Se han ejecutado diferentes proyectos tecnológicos por parte de instituciones del Estado y de ONGs. Asimismo, la comunidad campesina tiene sus propias iniciativas de adopción de nuevas tecnologías agropecuarias debido a que los proyectos ejecutados por el Estado no son suficientes.

En este contexto, es necesario que la academia centre sus investigaciones en las tecnologías ancestrales que aún no han sido reconocidas pero que son utilizadas por las comunidades campesinas. También investigaciones para solucionar los problemas cotidianos a los que se enfrenta el campesino, como plagas, malezas y técnicas de conservación de agua, suelo y vegetación. Asimismo, investigación de los productos potenciales que puedan ser comercializados a buen precio en los mercados locales y las ciudades.

Finalmente, las instituciones que ejecutan proyectos tecnológicos deben de tener presente que el reforzamiento de la organización comunal en la agricultura familiar es elemental, así como la distribución de los roles entre mujeres, varones y 
jóvenes tal que todos participen. Las capacitaciones en organización comunal, manejo de tecnologías modernas y temas comerciales son fundamentales para la sostenibilidad de los proyectos.

\section{Agradecimientos}

Las autoras manifiestan sus sinceros agradecimientos al Mag. Lic. Johnatan Vega Slee por sus sugerencias en las mejoras de la redacción del artículo.

\section{Referencias}

[1] FAO. Plataforma de conocimientos sobre agricultura familiar [Online]. Disponible en: https://www.fao.org/family-farming/

[2] J.P. Aryal, D. B. Rahut, G. Thapa and F. Simtowe, "Mechanisation of small-scale farms in South Asia: Empirical evidence derived from farm households survey", Technology in Society, vol. 65, pp. 1-14, May 2021.

[3] Y. Gong, J. Li and Y. Li, "Spatiotemporal characteristics and driving mechanisms of arable land in the Beijing-Tianjin-Hebei region during 1990-2015", Socio-Economic Planning Sciences, vol. 70, June 2020

[4] G. P. Paudel, D. B. KC, D. B. Rahut, S. E. Justice, and A. J. McDonald, "Scaleappropriate mechanization impacts on productivity among smallholders: Evidence from rice systems in the mid-hills of Nepal", Land use Policy, vol. 85, pp. 104-113, June 2019.

[5] K. A. Mottaleb, T. J. Krupnik and O. Erenstein,"Factors associated with smallscale agricultural machinery adoption in Bangladesh: Census findings", Journal of Rural Studies, vol. 46, pp. 155-168, August 2016.

[6] G. P. Paudel, D. B. KC, D. B. Rahut, N. P. Khanal, S. E. Justice, and A. J. McDonald, "Smallholder Farmers' Willingness to Pay for Scale-Appropriate Farm Mechanization: Evidence from the Mid-Hills of Nepal", Technology in Society, vol. 59, November 2019.

[7] Y. Jiang, Y. Tang, H. Long and W. Deng, "Land consolidation: A comparative research between Europe and China", Land Use Policy, vol. 112, January 2022.

[8] H. Arita and K. Kimura, Land consolidation of paddy field, Japan: Association of Agriculture \& Forestry Statistics, 2003.

[9] J. Rolando, C. Turin, D. Ramírez, V. Mares, J. Monerris and R. Quiroz, "Key ecosystem services and ecological intensification of agriculture in the tropical high-Andean Puna as affected by land-use and climate changes", Agriculture, Ecosystems \& Environment, vol. 236, pp. 221-233, January 2017.

[10] C. Hammond, M. Cox and J.L. Bazo, "Pesticide lock-in in small scale Peruvian agriculture", Ecological Economics, vol. 129, pp. 72-81, September 2016.

[11] V. Carrion, P. Gallegos, V. Barrera, G.W. Norton and J. Alwang, "IPM Technologies for Potato Producers in Highland Ecuador", in Integrated Pest Management of Tropical Vegetable Crops, R. Muniappan, E.Heinrichs, Virginia: Springer, Dordrecht, 2016.

[12] M. De La Cruz and J. Dessein, "Beyond institutional bricolage: An 'intertwining approach' to understanding the transition towards agroecology in Peru", Ecological Economics, vol. 187, September 2021.

[13] M. Garfí, J. Martí-Herrero, A. Garwood and I. Ferrer, "Household anaerobic digesters for biogas production in Latin America: A review", Renewable and Sustainable Energy Reviews, vol. 60, pp. 599-614, July 2016.

[14] L. Ferrer-Martí, I. Ferrer, E. Sánchez and M. Garfí, "A multi-criteria decision support tool for the assessment of household biogas digester programmes in rural areas. A case study in Peru", Renewable and Sustainable Energy Reviews, vol. 95, pp. 74-83, November 2018.

[15] J. Cevallos-Sierra and J. Ramos-Martin, "Spatial assessment of the potential of renewable energy: The case of Ecuador", Renewable and Sustainable Energy Reviews, vol. 81, pp. 1154-1165, January 2018.

[16] Congreso (2015, Noviembre 03). Ley 30355 [Online]. Disponible en: https://busquedas.elperuano.pe/normaslegal es/ley-de-promocion-y-desarrollo-de-laagricultura-familiar-ley-n-30355-1307649-2/

[17] MINAGRI (2019, Noviembre 19). Decreto Supremo $\quad \mathrm{N}^{\circ}$ 007-2019-MINAGRI que aprueba el Plan Nacional de Agricultura Familiar 2019-2021.[Online]. Disponible en: https://www.gob.pe/institucion/midagri/norm as-legales/357293-007-2019-minagri

[18] C. Alva, B. Noriyuki and J. Velásquez, "Costos y rendimientos en la labranza convencional y mecanizada de la agricultura familiar en la comunidad campesina Barrio 
Bajo de Matucana", ECIPerú, vol. 12, no. 2, pp.135-142, diciembre 2015.

[19] J. Velásquez, Recuperación de andenes y manejo del agua en la comunidad campesina Barrio Bajo de Matucana, Lima: Banco Interamericano de Desarrollo (BID), Agrorural, 2013.

[20] MEF. Banco de Inversiones. Consulta Avanzada de Inversiones. [Online]. Available:

http://ofi5.mef.gob.pe/inviertePub/ConsultaP ublica/ConsultaAvanzada

[21] ANA (2010, diciembre). Estudio hidrológico y ubicación de la red de estaciones hidrométricas en la cuenca del río Rímac. Vol. 1 [Online] Disponible: http://sigrid.cenepred.gob.pe/docs/PARA\%2 OPUBLICAR/ANA/Estudio_hidrologico_Cue nca_Rimac_volumen_I_texto_final_2010.pdf

[22] INEI (2017). Censos Nacionales de Población y Vivienda 2017. [Online] Disponible en: https://censos2017.inei.gob.pe/redatam/

[23] IBC and CEPES, Directorio de Comunidades campesinas del Perú. Lima: BNP, 2016.

[24] INEI (2012). IV Censo Nacional Agropecuario. [Online] Disponible en: http://censos.inei.gob.pe/Cenagro/redatam

[25] MIDAGRI. Estadística agropecuaria, en Sistema Integrado de Estadística Agraria (SEIA-BI) [Online]. Disponible en: https://siea.midagri.gob.pe/portal/siea_bi

[26] MIDAGRI (2020, diciembre 21). Resolución Ministerial $\quad \mathrm{N}^{\circ}$ 0322-2020-MIDAGRI. Oficializan el Mapa Nacional de Superficie Agrícola del Perú [Online] Disponible en: https://www.gob.pe/institucion/midagri/norm as-legales/1433393-0322-2020-midagri

[27] MIDAGRI (2021, enero). Andenes para la vida. Inventario y caracterización de andenes en los andes tropicales del Perú (Primera edición) [Online]. Disponible en: https://www.agrorural.gob.pe/wpcontent/uploads/2021/02/Libros-Andenespara-la-vida-PDF.pdf

[28] MINAGRI (2018, julio 18). Resultado de la Encuesta de la Campaña Agrícola Agosto 2018- Julio 2019 [Online]. Disponible en: https://www.gob.pe/institucion/midagri/infor mes-publicaciones/354146-resultado-de-laencuesta-de-la-campana-agricola-agosto2018-julio-2019

[29] MINAGRI (2019, octubre 17). Resultado de la Encuesta de la Campaña Agrícola Agosto 2019 - Julio 2020 [Online]. Disponible en: https://www.gob.pe/institucion/midagri/infor mes-publicaciones/354122-resultado-de-laencuesta-de-la-campana-agricola-agosto2019-julio-2020

[30] C. Muñoz, Cómo elaborar y asesorar una investigación de tesis. Segunda edición. México: Pearson Educación, 2011

[31] J. Benites (entrevista personal), 2021

[32] M. Epiquién (entrevista personal), 2021

[33] M. Varas (entrevista personal), 2021.

[34] F. Guevara (entrevista personal), 2021

[35] E. Garner and A. P. de la O Campos, Identifying the "family farm": An informal discussion of the concepts and definitions, Rome: FAO, 2014.

[36] I. Medina (entrevista personal), 2021

[37] R. Melo (entrevista personal), 2021

[38] E. Schatzberg, Technology: Critical History of a Concept, Chicago and London: The University of Chicago Press, 2018.

[39] C. Alva, "Proyectos de inversión sobre técnicas de comunidades campesinas alto andinas para el desarrollo de ciudades resilientes" en $12^{\circ}$ Simposio Internacional de Gestión del Riego de Desastres, Lima, PE, 2018, pp. 173-175.

[40] C. Milla. Génesis de la Cultura Andina. Sexta edición. Lima: BNP, 2011.

[41] C. Felipe-Morales (entrevista personal), 2021

catherine.alva.ru.mo@gmail.com melchorita.castro@unmsm.edu.pe merely.tumbalobos@unmsm.edu.pe 